\title{
Serotonin Transporter Defect Disturbs Structure and Function of the Auditory Cortex in Mice
}

\author{
Wenlu Pan ${ }^{1,2}$, Jing Pan ${ }^{3,4}$, Yan Zhao', Hongzheng Zhang ${ }^{3,4 *}$ and Jie Tang ${ }^{1,3,4,5 *}$ \\ ${ }^{1}$ Department of Physiology, School of Basic Medical Sciences, Southern Medical University, Guangzhou, China, ${ }^{2}$ Functional \\ Nucleic Acid Basic and Clinical Research Center, Department of Physiology, School of Basic Medical Sciences, Changsha \\ Medical College, Changsha, China, ${ }^{3}$ Department of Otolaryngology Head and Neck Surgery, Zhujiang Hospital, Southern \\ Medical University, Guangzhou, China, ${ }^{4}$ Hearing Research Center, Southern Medical University, Guangzhou, China, ${ }^{5}$ Key \\ Laboratory of Mental Health of the Ministry of Education, Southern Medical University, Guangzhou, China
}

\section{OPEN ACCESS}

Edited by:

Ke Liu,

Beijing Friendship Hospital, Capital Medical University, China

Reviewed by:

Meagan A. Quinlan, University of Washington,

United States

Ling Qin,

China Medical University, China

*Correspondence: Hongzheng Zhang redtrue@smu.edu.cn Jie Tang

jietang@smu.edu.cn

Specialty section: This article was submitted to Auditory Cognitive Neuroscience, a section of the journal

Frontiers in Neuroscience

Received: 30 July 2021 Accepted: 07 September 2021 Published: 06 October 2021

Citation:

Pan W, Pan J, Zhao Y, Zhang H and Tang J (2021) Serotonin Transporter Defect Disturbs Structure and Function of the Auditory Cortex in Mice. Front. Neurosci. 15:749923.

doi: 10.3389/fnins.2021.749923
Serotonin transporter (SERT) modulates the level of 5-HT and significantly affects the activity of serotonergic neurons in the central nervous system. The manipulation of SERT has lasting neurobiological and behavioral consequences, including developmental dysfunction, depression, and anxiety. Auditory disorders have been widely reported as the adverse events of these mental diseases. It is unclear how SERT impacts neuronal connections/interactions and what mechanism(s) may elicit the disruption of normal neural network functions in auditory cortex. In the present study, we report on the neuronal morphology and function of auditory cortex in SERT knockout (KO) mice. We show that the dendritic length of the fourth layer (L-IV) pyramidal neurons and the second-to-third layer (L-II/III) interneurons were reduced in the auditory cortex of the SERT KO mice. The number and density of dendritic spines of these neurons were significantly less than those of wild-type neurons. Also, the frequencytonotopic organization of primary auditory cortex was disrupted in SERT KO mice. The auditory neurons of SERT KO mice exhibited border frequency tuning with highintensity thresholds. These findings indicate that SERT plays a key role in development and functional maintenance of auditory cortical neurons. Auditory function should be examined when SERT is selected as a target in the treatment for psychiatric disorders.

Keywords: serotonin transporter, auditory cortex, dendritic spines, tonotopic map, hearing disorder

\section{INTRODUCTION}

Serotonin, one of the most widely spread neurotransmitters in the central nervous system, has been known to play a critical role in brain morphogenesis and functions (Azmitia, 2001; Gaspar et al., 2004). The defects of serotonergic neurons are related to many psychiatric disorders, including depression, anxiety, and autism spectrum disorder (ASD) (Simpson et al., 2011). Acting as a key regulator of serotonergic activity, the serotonin transporter (SERT) is usually selected as the target of antidepressant treatments. SERT represents a potential mediator for anxiety- and depressionrelated behaviors. However, chronic exposure to selective serotonin reuptake inhibitors (SSRIs) was reported to elicit hearing disorders, such as tinnitus (Kehrle et al., 2015; Pattyn et al., 2016), auditory hallucinations (Kogoj, 2014), and hearing loss (Blazer and Tucci, 2018). 
However, how the functions of SERT affect the auditory system remains unclear. Both increases and decreases in serotonin levels during early development have been found to impair the formation and function of the primary somatosensory cortex of rodents (Cases et al., 1996; Persico et al., 2000; Jennings et al., 2006). Pre- and post-natal exposure to the SSRI disturbed the chemoarchitecture of the mouse auditory cortex (AC) and resulted in ASD-like behavior (Simpson et al., 2011). Our previous study found that both SSRI treatment and SERT knockout $(\mathrm{KO})$ did not change the auditory brain responses but abolished the auditory mismatch negativity in adult animals (Pan et al., 2020). These investigations suggest that SERT may affect the auditory functions by manipulating the serotonin level and serotonergic neurons of AC.

In rodents, 2 weeks after birth, layer IV afferent neurons in the primary visual, auditory, and somatosensory cortex are innervated by aggregates of serotonin-containing processes (D’Amato et al., 1987; Blue et al., 1991). At present, the morphological and especially the functional implications of SERT in $\mathrm{AC}$ remain unclear. In the present study, we examined the fine dendritic structure of the neurons in primary AC. We found that although expression of SERT is low in the AC, both pyramidal neurons and interneurons in SERT KO mice showed significant reductions in dendritic length, the number, and density of dendritic spines than did wild-type (WT) mice. The electrophysiological features of AC were also impaired by the SERT deficit. These results suggest that SERT plays a key role in development and functional maintenance of AC. This may explain the observed hearing disorders in patients utilizing drugs that target SERT, such as SSRIs, in the treatment for psychiatric disorders.

\section{MATERIALS AND METHODS}

All the animal experiments involved in this study were approved by the Institutional Animal Care and Use Committee (IACUC) of Southern Medical University. The principles formulated by the Animal Care Committee of Southern Medical University were followed throughout the experiment.

\section{Subjects}

SERT KO mice were derived from the Jackson Laboratory (Stock No. 008,355) and backcrossed with C57BL/6J background mice (Bengel et al., 1998). Polymerase chain reaction (PCR) protocol used for genotyping SERT KO mice has been reported in our previous literature (Pan et al., 2020). Male and female mice aged 2-4 months and weighting 20-26 g were employed in our test. Animals were housed in a room maintained at $22^{\circ} \mathrm{C}\left( \pm 2^{\circ} \mathrm{C}\right)$ and kept on a 12:12 light/dark cycle with lights on at 8:00 a.m.

\section{Immunohistochemistry}

In this study, adult $\mathrm{C} 57 \mathrm{BL} / 6 \mathrm{~J}$ mice were deeply sedated with pentobarbital sodium $(50 \mathrm{mg} / \mathrm{kg})$ administered via intraperitoneal injection. The mice were perfused intravascularly via the left ventricle with phosphate-buffered saline (PBS; $\mathrm{pH}$ 7.4), followed by a fixative, viz., $4 \%(\mathrm{w} / \mathrm{v})$ paraformaldehyde. The mice were decapitated; the brain and cochleae were harvested and fully fixed in $4 \%$ paraformaldehyde, at $4^{\circ} \mathrm{C}$ overnight. Then the brain was sectioned on a freezing microtome to a thickness of $50 \mu \mathrm{m}$. The cochleae were washed in PBS, then placed into $10 \%$ ethylenediaminetetraacetic acid (EDTA) decalcifying solution, and replaced with fresh decalcifying solution every day for 6-8 days. Decalcification is terminated when the cochleae were transparent and elastic. Then the cochleae were sliced up parallel to the modiolar plane of the cochlea, and thickness of the slices was $10 \mu \mathrm{m}$. Brain and cochleae tissue samples were permeabilized in $0.3 \%$ Triton X-100 (Gibco, Grand Island, NY, United States) for $1 \mathrm{~h}$ and immunoblocked with a solution of $10 \%$ goat serum albumin for an additional hour. The specimens were incubated overnight at $4^{\circ} \mathrm{C}$ with SERT antibody (Millipore, Billerica, MA, United States; cat. no. 2828614) diluted in 10\% goat serum albumin. After several washes in PBS, the specimens were then incubated with the Alexa-Fluor-488-conjugated secondary antibody at a concentration of 1:1,000 for $1 \mathrm{~h}$ at room temperature. To assign neurons, sections were counterstained for $20 \mathrm{~min}$ at room temperature with a fluorescent dye NeuroTrace 530/615 (1:100, Invitrogen, Carlsbad, CA, United States). Samples were then washed with PBS for three times and examined by using a Nikon confocal microscope (Nikon Instruments Inc., Melville, NY, United States).

\section{Golgi Staining}

Golgi staining was used to visualize the dendritic branching complexity and spines of the neurons in the mice. Mice were anesthetized with $10 \%$ chloral hydrate. The brains were taken after being fully infused with $0.9 \%$ saline and $4 \%$ paraformaldehyde, successively. Then, the brains were immersed in Golgi-Cox solution (Glaser and Loos, 1981) and stored at room temperature for 2 weeks in the dark. Next, the brains were transferred to a $30 \%$ sucrose solution and dehydrated at $4^{\circ} \mathrm{C}$ for 2-5 days, avoiding light. The brain tissues were completely coated with OCT embedding agent (Tissue-Tek 4583; Sakura Finetek United States, Inc., Torrance, CA, United States). The $100-\mu \mathrm{m}$-thick sections were prepared on gelatin-coated slides in a coronal plane parallel to the base and left to air-dry away from light for 2 days before being processed for Golgi-Cox impregnation. The brain slides were put into a special opaque staining box, and Golgi-Cox staining was performed as the literature (Zhang et al., 2009).

\section{Sholl's Analysis}

Dendrites in each of the selected neurons were quantitatively analyzed using Sholl's concentric circle method (Sholl, 1953). The neurons were selected from layers IV and II/III in AC using the following criteria: (1) the cell body was in the subregion of interest, (2) the staining of the branches was efficient and complete throughout the length, and (3) the branches were isolated from their neighbors. A series of concentric rings, spaced $20 \mu \mathrm{m}$ apart, were placed over the neuron and centered on the cell body, and the number of dendrites intersecting each circle in the series of concentric circles was counted blind to the experimental conditions to estimate the total dendritic length, branch points, and dendritic complexity. 


\section{Dendritic Spine Density Analysis}

Spine analyses were conducted blind to the experimental conditions on coded Golgi impregnated brain sections containing the AC. Spines were examined on dendrites of pyramidal neurons and inter neurons. Briefly, all protruding dendritic spines were counted on per $25-\mu \mathrm{m}$ dendritic segments. Spine density was expressed as the number of spines per $25 \mu \mathrm{m}$. Two to three dendritic segments were analyzed per neuron. Only intact, properly stained, and unbranched dendritic segments were included in the analyses. To acquire images for spine analysis, the dendritic segments were imaged under bright field illumination on a Zeiss Axioimager microscope (Carl Zeiss, Oberkochen, Germany) with a $63 \times$ oil immersion objective.

\section{Recording in the Primary Auditory Cortex (Al)}

WT and SERT KO mice weighing 20-26 g were anesthetized by pentobarbital sodium $(30 \mathrm{mg} / \mathrm{kg}$, i.p.), followed by atropine sulfate $(0.25 \mathrm{mg} / \mathrm{kg}$, i.h.) to prevent asphyxia. The level of anesthesia was maintained by additional dosages of sodium pentobarbital $(30 \mathrm{mg} / \mathrm{kg}$, i.p.) administered approximately every $40 \mathrm{~min}$ throughout the physiological experiments. Under anesthesia, the mouse's head was fixed in a head holder by rigidly clamping on a nail about $1.5 \mathrm{~cm}$ long fixed on the surface of the skull vertically with dental cement (Tang et al., 2008; Tang and Suga, 2009).

The scalp, muscles, and soft tissues of the skull were then removed; an opening above the left $\mathrm{AC}$ was made using a dental driller; and the dura was gently removed. The anatomical location of $\mathrm{AI}$ in $\mathrm{AC}$ was marked according to the brain map (bregma $-2.7 \mathrm{~mm}$, left/right of the midline $3.5 \mathrm{~mm}$ ) (Umbricht et al., 2005), and the size of the parietal open window was $0.2 \times 0.2 \mathrm{~mm}^{2}$. The cortex was maintained under artificial cerebrospinal fluid to prevent desiccation. The mouse was placed on a feedback-controlled heating pad to maintain its body temperature at $37^{\circ} \mathrm{C}$. All electrophysiological experiments were performed in a soundproof and echo-attenuated chamber.

Microelectrodes with a $\sim 1-\mu \mathrm{m}$ tip diameter (7-12 $\mathrm{M} \Omega$, filled with $3 \mathrm{~mol} / \mathrm{L}$ of $\mathrm{KCl}$ ) were used for recordings. At every recording site, the microelectrode was lowered orthogonally into the cortex to depths of 200-375 $\mu \mathrm{m}$ (layers II/III) or 475-600 $\mu \mathrm{m}$ (layers IV/V), where the evoked spikes of a neuron or a small cluster of neurons were collected. After the best frequency (BF) of neurons was found at a recording point once, the electrode was moved toward the rostral or caudal side to the next point 200-300 $\mu \mathrm{m}$ away from the previous recording point, and the same measurement was repeated. Complete examinations were repeated in this way until no response at the two adjacent voiceinduced recording sites was observed in any direction.

Pure tones were generated and played with a TDT 3 system (Tucker-Davis Technologies, Alachua, FL, United States) for auditory mapping. A real-time processor (RP2.1) and a program written in RPvdsEx software were used to synthesize the sound signals. Sound intensity was adjusted by an attenuator (PA5). The synthesized sound signal was amplified by an electric driver (ED1) with an open-field speaker (ES1). Before the OpenEx software (sampling rate $=25 \mathrm{kHz}$ ) recording, the speakers were calibrated with 1/8- and 1/4-inch microphones (Brüel and Kjaer 4138, 4135, Naerum, Denmark). Neural signals were amplified $10,000 \times$ using a digital amplifier (RA16) with a $0.3-$ to $3-\mathrm{kHz}$ filter and monitored online by a software Brainware (TuckerDavis Technologies). Frequency-intensity receptive fields (RFs) were reconstructed in detail by presenting pure tones $(50-\mathrm{ms}$ duration, 5-ms ramps) of six frequencies $(2-32 \mathrm{kHz})$ at nine sound intensities [0-90 dB sound pressure level (SPL), in 10-dB increments] at a rate of two stimuli per second. The tones were presented in a random, interleaved sequence.

To generate the cortical map, we used Matlab functions to create colored polygons, constructed by connecting a record point and intermediate points between four and six adjacent record points (Bao et al., 2003). The electrode penetration point was located in its center, and each polygon was the BF with responsive neurons at this point. In the topological diagram, the RF of auditory neurons in area AI was continuous, single-peaked, and $\mathrm{V}$-shaped. Moreover, the $\mathrm{BF}$ of the neurons in this region was tonotopically organized, with high frequencies in the rostrally and low frequencies in the caudally.

\section{Data Processing and Statistical Analysis}

We defined the minimum threshold (MT) and BF to enable neurons to respond to the minimum stimulus sound intensity at the corresponding sound frequency when firing maximum number of spikes at this site. The BF of the cortical region $\mathrm{AI}$ was defined as the frequency at the tip of the tuning curve, that is, the sound frequency corresponding to the MT of the neuron at this place. The sharpness of the frequency-tuning curve, defined for all recording sites, was represented by the value Q30 (Tang et al., 2012). Q30 was equal to the BF divided by the bandwidth value of $30 \mathrm{~dB}$ above the MT within the frequency-intensity RF range. The larger the Q30, the sharper the tuning curve representing the neurons, and the better the frequency selectivity of the neurons. A customized MatLab program (MathWorks, Natick, MA, United States) was used to analyze and plot tonotopic map of A1. Tonotopic index (TI) was used to evaluate the tonotopic organization by following the methods described in previous studies (Zhang et al., 2001; Bao et al., 2004).

We used SPSS 20 software (IBM, Armonk, NY, United States) to perform statistical analysis. The two-tailed $t$-tests (for unpaired comparisons) and two-way ANOVA (for multiplegroup comparisons) were used to test for significant differences between groups. Statistical significance was defined as $p<0.05$. GraphPad Prism 7 (GraphPad Software, San Diego, CA, United States) was used for plotting.

\section{RESULTS}

By using immunostaining, the expression of SERT in the auditory neural system was examined systematically. As shown in Figure 1, no robust SERT expression was detected in the cochlea (spiral ganglion neurons; Figure 1A), auditory brainstem (cochlear nuclei, superior olivary complex, and inferior colliculus; Figures 1B-D), or auditory thalamus (medial 

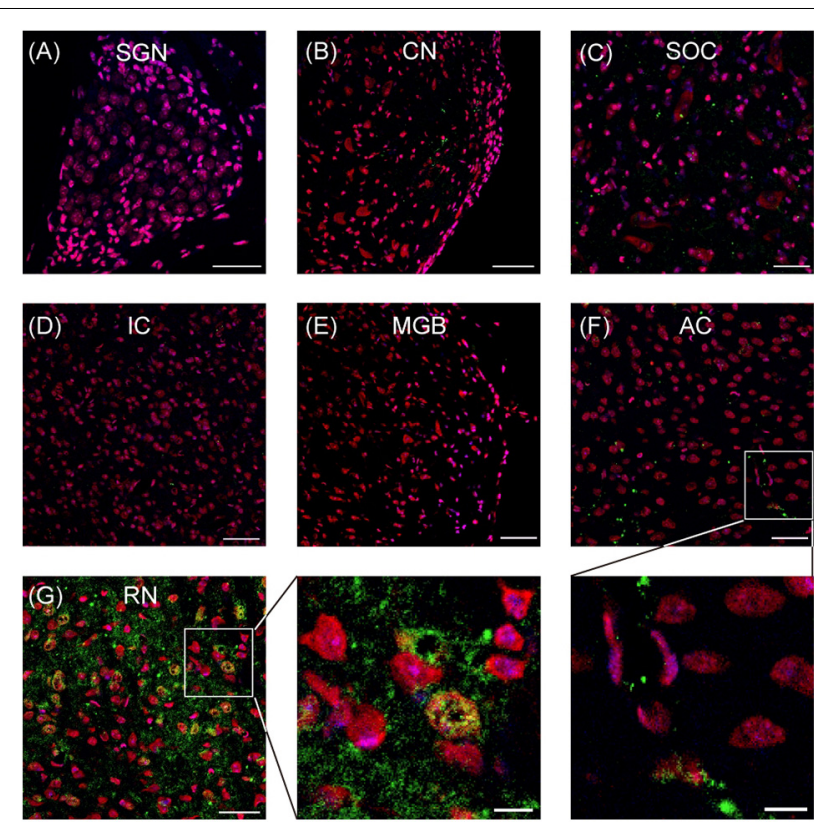

FIGURE 1 | Representative images show the serotonin transporter (SERT) expression in different regions of auditory system. (A-G) Confocal images show the SERT (green) expression in the spiral ganglion neurons (SGN), cochlear nuclei (CN), superior olivary complex (SOC), inferior colliculus (IC), medial geniculate body (MGB), auditory cortex (AC), and raphe nuclear complex (RN). Insets are the magnified images of the boxes in (F,G). The soma and nucleus of neurons are colored in red (Nissl) and blue (DAPI), respectively. SERT is colored in green. Scale bars: $50 \mu \mathrm{m}$ for $(\mathbf{A}-\mathbf{G})$ and $10 \mu \mathrm{m}$ for insets.

geniculate body; Figure 1E). Hence, we examined the SERT expression in the primary AC. Our data from AC exhibit sporadic spread of SERT immunoreactive puncta, which represents the SERT immunoreactive fibers (Figure 1F). However, the SERT immunoreactive puncta was usually detected around the soma of cortical neurons (inset of Figure 1F) with a much lower density than the expression in the raphe nuclear complex (Figure 1G). This result suggests that $\mathrm{AC}$ neurons receive sparse serotonergic projections, if any.

To determine the roles of these serotonergic projections, the morphology of AC neurons from SERT KO mice was examined in comparison with that of WT mice. Our data show that for pyramidal cells in layer IV, the number of intersections and the total length of dendrites were significantly reduced in both apical and basal dendrites of the SERT KO AC neurons (Figure 2). Moreover, the reduction of dendrites was also found in layer II/III interneurons of SERT KO AC (Figures 2F,G).

Most excitatory synaptic connections occur on dendritic spines. Spines can individually detect the temporal coincidence of pre- and post-synaptic activities and thus serve as basic functional units of neuronal integration (Gray, 1959; Yuste and Denk, 1995; Spacek and Harris, 1998; Arellano et al., 2007). To further determine whether synaptic function at neuronal junctions was altered in SERT KO mice, we looked at the morphologic structure of the dendritic spines of AC neurons. We measured the number and density of total dendritic spines in layer IV pyramidal cells and layer II/III interneurons from the AC of WT and SERT KO mice (Figure 3A). In apical dendrites, the total number of dendritic spines of layer IV pyramidal cells was less in SERT $\mathrm{KO}$ mice [vs. WT, $F(1,159)=252.5, p<0.0001$, two-way ANOVA]. Significant reduction was observed at a distance of 25-125 $\mu \mathrm{m}$ from cell bodies (vs. WT, $p<0.001$, two-tailed $t$-test; Figure 3B). The mean density of apical dendritic spines reduced significantly in SERT KO mice (vs. WT, $p<0.0001$, two-tailed $t$-test; Figure 3E). The total number of basal dendritic spines of layer IV pyramidal cells was also reduced in SERT KO mice [vs. WT, $F(1,356)=109.6, p<0.0001$, two-way ANOVA] and significantly reduced at a distance of $25-150 \mu \mathrm{m}$ from cell bodies (vs. WT, $p<0.001$, two-tailed $t$-test; Figure $3 \mathrm{C}$ ). Meanwhile, the mean density of basal dendritic spines reduced significantly in SERT KO mice (vs. WT, $p<0.0001$, twotailed $t$-test; Figure 3F). However, the total number of dendritic spines in layer II/III interneurons in SERT KO mice was not significantly different [vs. WT, $F(1,292)=10.93$, $p=0.011$, twoway ANOVA]. The density was only found reduced at a distance of 25-75 $\mu \mathrm{m}$ from cell bodies (vs. WT, $p<0.05$, two-tailed $t$-tests; Figure $3 \mathrm{D})$. The mean density of dendritic spines was also significantly reduced in SERT KO mice (vs. WT, $p<0.0001$, two-tailed $t$-test; Figure 3G). Together with the results of our Sholl's analysis, these morphological data implied that synaptic transmission between the subcortical and cortical neurons, as well as the neurons within the AC, might be weakened in the auditory system of SERT KO mice. These defects may affect the functions of SERT KO AC neurons.

Electrophysiological experiments were conducted to investigate the functional alteration of auditory neurons in primary AC (AI) of SERT KO mice. Previous studies have found that neurons at different locations within the same layer of AI respond to different frequencies (Kelly and Sally, 1988; Kaas et al., 1999; Zhang et al., 2001; de Villers-Sidani et al., 2007). Therefore, we measured the "frequency-tonotopic map" of neurons in the AI region of cortex. A total 246 sites and 243 sites were recorded from the auditory cortical area of WT and SERT KO mice, respectively. Normally in WT mice, high-frequency sensitive neurons are located in the rostral sites of the AI region, and low-frequency sensitive neurons are located in caudal side (Stiebler et al., 1997).

As shown in the representative data in Figure 4A, the BFs of AC neurons were distributed regularly from the rostral to caudal sites, forming a compact and ordered "tonotopic map" in WT mice. However, for AC neurons of SERT KO mice, their frequency selectivity did not show a systematic organization, although the neurons that respond to high frequencies were still mostly located to the rostral side. We further analyzed the frequency range and the total area of the AI region. No statistical difference was found between SERT KO and WT mice ( $p>0.05$, two-tailed $t$-test; Figures 4B,C). The BFs of all recorded sites were plotted against a normalized AC axis (Figure 4D). The distribution of BFs was quantified with the TI, which was significantly increased in SERT KO mice $(p<0.01$, two-tailed $t$-test). These results showed that a disrupted tonotopic map was found in the AC of SERT KO mice, suggesting that SERT may mainly affect the neuron's tuning property. 


\section{A}

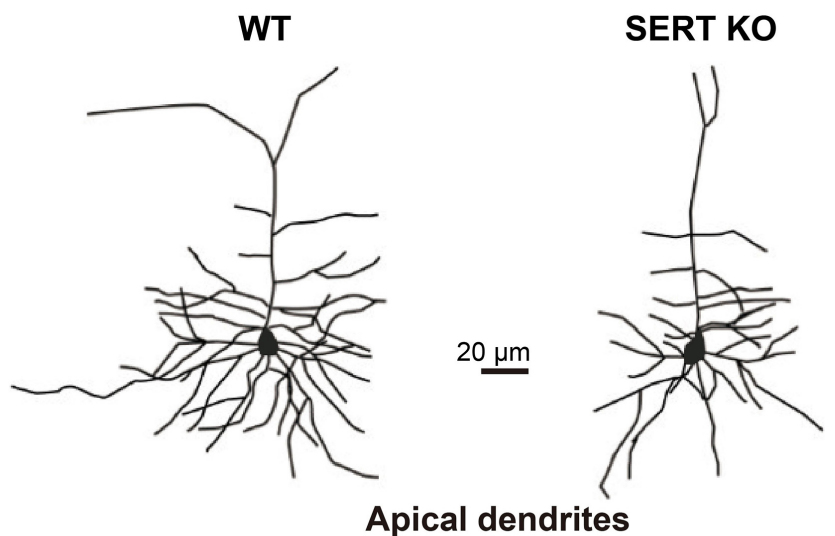

B

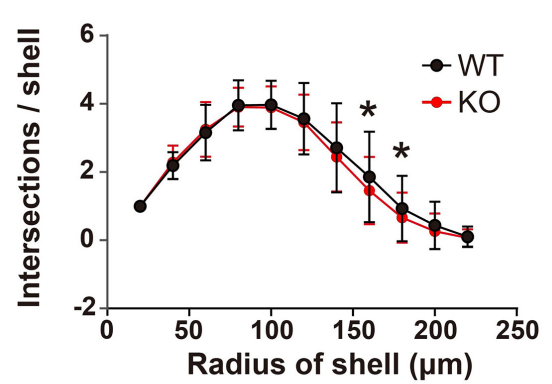

C

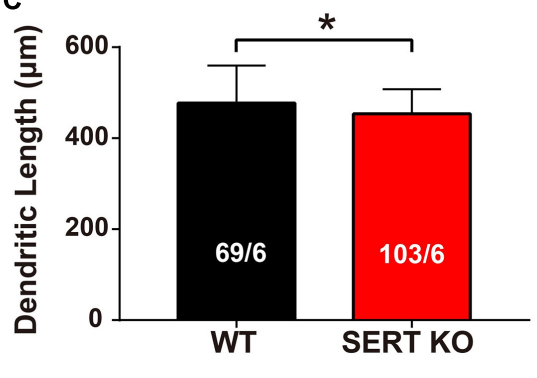

D

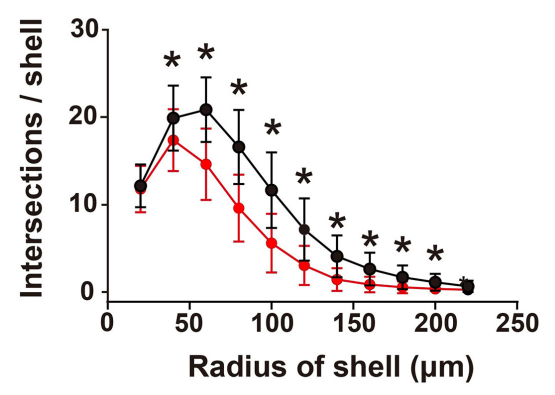

Basal dendrites

E

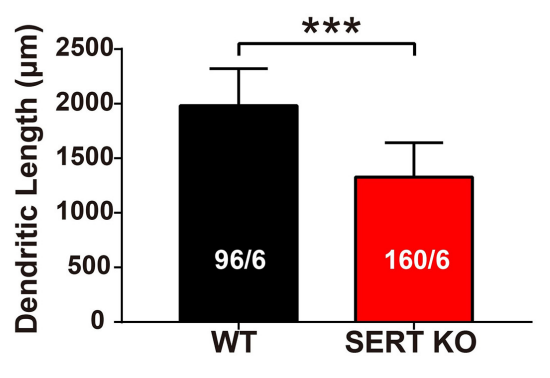

Inter neuron

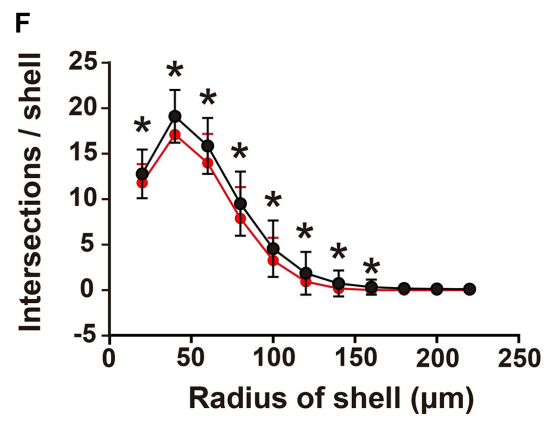

G

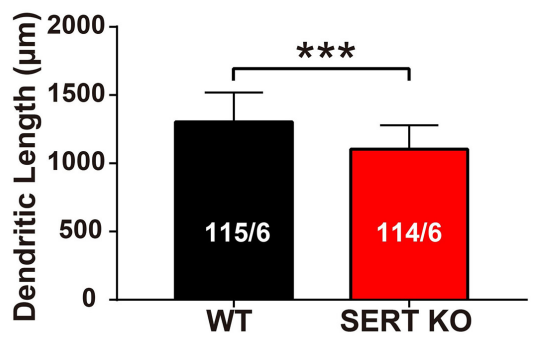

FIGURE 2 | Serotonin transporter (SERT) knockout (KO) degrades the dendrites of layer IV pyramidal neurons and layer II/III interneurons in auditory cortex. (A) Representative dendritic morphology of layer IV pyramidal cells in auditory cortex from wild-type (WT) and SERT KO mice. Scale bar: $20 \mu \mathrm{m}$. (B,C) Comparing the number of intersections and the total length of the apical dendrites of layer IV pyramidal cells in WT mice with SERT KO mice. (D,E) Comparing the number of intersections and the total length of the basal dendrites of layer IV pyramidal cells in WT mice with SERT KO mice. (F,G) The number of intersections and the total length of the dendrites of layer II/III interneurons were significantly reduced in SERT KO mice, compared with WT mice. The number of intersections of dendrites was measured with 20- $\mu \mathrm{m}$ concentric spheres centered on the soma by Sholl's analysis. The numbers in the column indicate the numbers of neurons/numbers of animals analyzed. Data are presented as means \pm SD. ${ }^{*} p<0.05 ;{ }^{\star \star \star} p<0.001$, Student's $t$-tests and two-way ANOVA. 
A

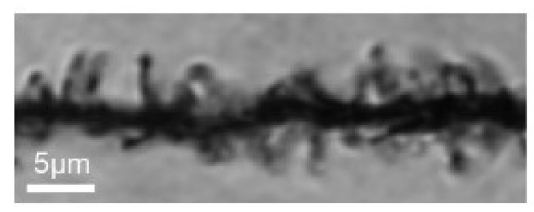

SERT KO

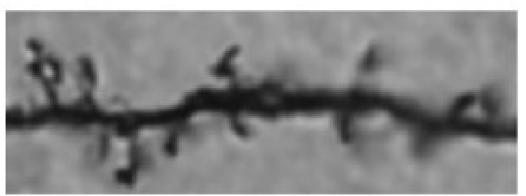

B

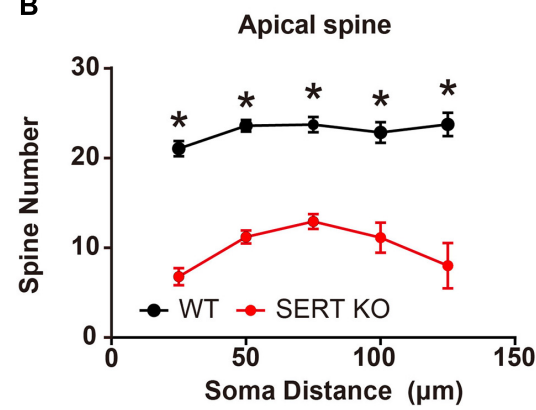

E

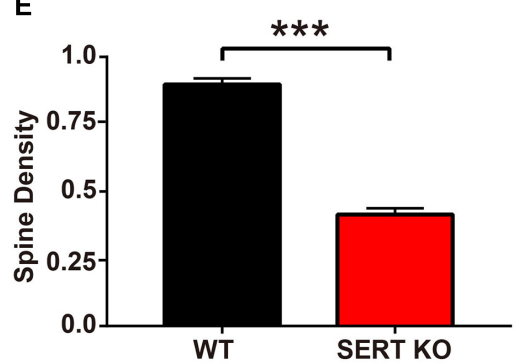

C

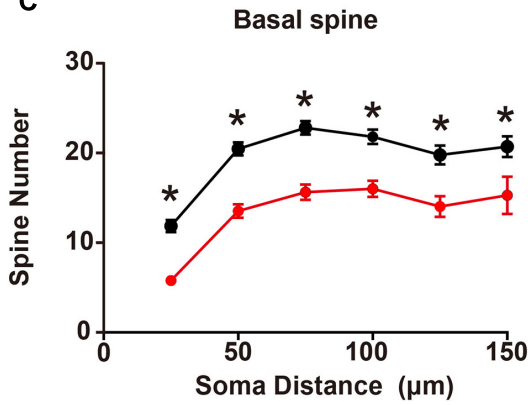

F

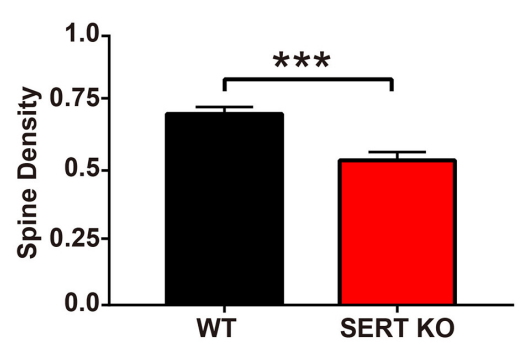

D

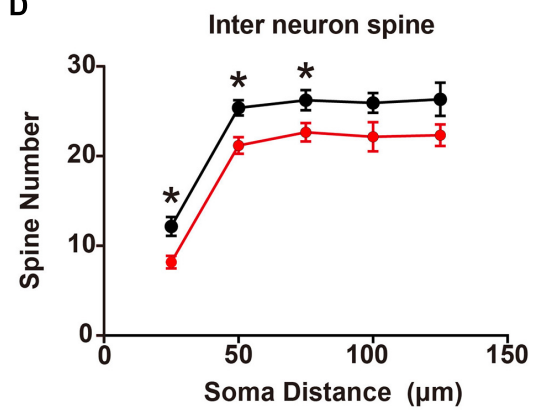

G

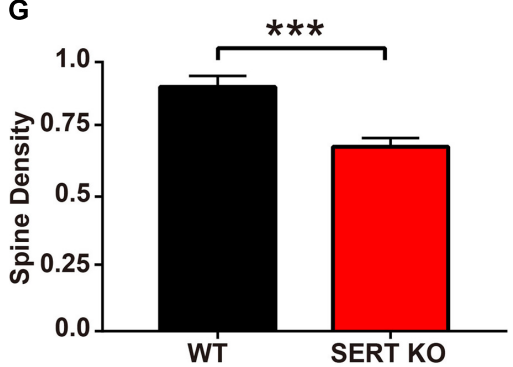

FIGURE 3 | Dendritic spines density decreased in serotonin transporter (SERT) knockout (KO) mice. (A) Representative photomicrographs of dendritic spines of basal dendrites in layer IV pyramidal neurons from wild-type (WT) and SERT KO mice. (B,C) Comparing the total number of apical and basal dendritic spines at a distance of per $25 \mu \mathrm{m}$ from the cell body of layer IV pyramidal cells in WT mice with SERT KO mice, respectively. (D) Comparing the total number of dendritic spines at a distance of per $25 \mu \mathrm{m}$ from the cell body of layer II/III interneurons in WT mice with SERT KO mice. (E-G) The dendritic spine densities of apical and basal dendrites in layer IV pyramidal neurons and in layer II/III interneurons of the auditory cortex. Data are presented as means \pm SD. $n=6$ for each group. ${ }^{*} p<0.05$, ${ }^{\star \star \star} p<0.001$, Student's $t$-test and two-way ANOVA. Scale bar: $5 \mu \mathrm{m}$.

We then measured the $\mathrm{BF}$ and the MT of AC neurons recorded from SERT KO and WT mice, by which the frequency selectivity and sound sensitivity of neurons were compared. The BFs and MTs of SERT KO and WT neurons were pooled in Figures 5A,B, respectively. Interestingly, although no change was found in BFs, the MTs of SERT KO neurons were significantly elevated. The MTs of WT neurons were generally below $40 \mathrm{~dB}$ SPL with an average of $27.09 \pm 12.81 \mathrm{~dB}$ SPL. However, the MTs of SERT KO neurons were significantly higher with an average of $53.85 \pm 11.33 \mathrm{~dB}$ SPL $(p<0.0001$, two-tailed $t$-test; Figure 5C). These data suggest that individual neurons in SERT KO mice were significantly less sensitive to sound stimulation.

Auditory neurons respond not only to their most sensitive frequency (i.e., BF) but also to other frequencies of sound. One of the most important functions of auditory neurons is that they can selectively respond to sound within a range of frequencies. In WT mice, individual neurons respond to sound frequencies in addition to the BF at sound levels above MT. These frequency-threshold intensity points formed a "V-shaped" frequency-tuning curve (Figure 6A). In SERT KO mice, the frequency-tuning curves of cortical neurons have much wider bandwidth (Figure 6B). To evaluate the frequency selectivity of a single neuron, we used "Q30" value to analyze the changes in frequency selectivity of AC neurons. The higher the value of Q30, the sharper the frequency-tuning curve of the neuron, and the better the frequency selectivity. For AC neurons of WT mice, their Q30 values mostly ranged from 1 to 2.5, with an average of $1.78 \pm 0.91$ (Figures 6C,D). But in SERT KO mice, Q30 values of most AC neurons were below 1 $(0.65 \pm 0.27$, mean $\pm \mathrm{SD}$; Figures 6C,D). This result suggested that $\mathrm{AC}$ neurons in SERT KO mice have less selectivity to sound frequency.

\section{DISCUSSION}

Our results of immunohistochemical study in the whole auditory system showed that SERT was rarely expressed in the auditory 

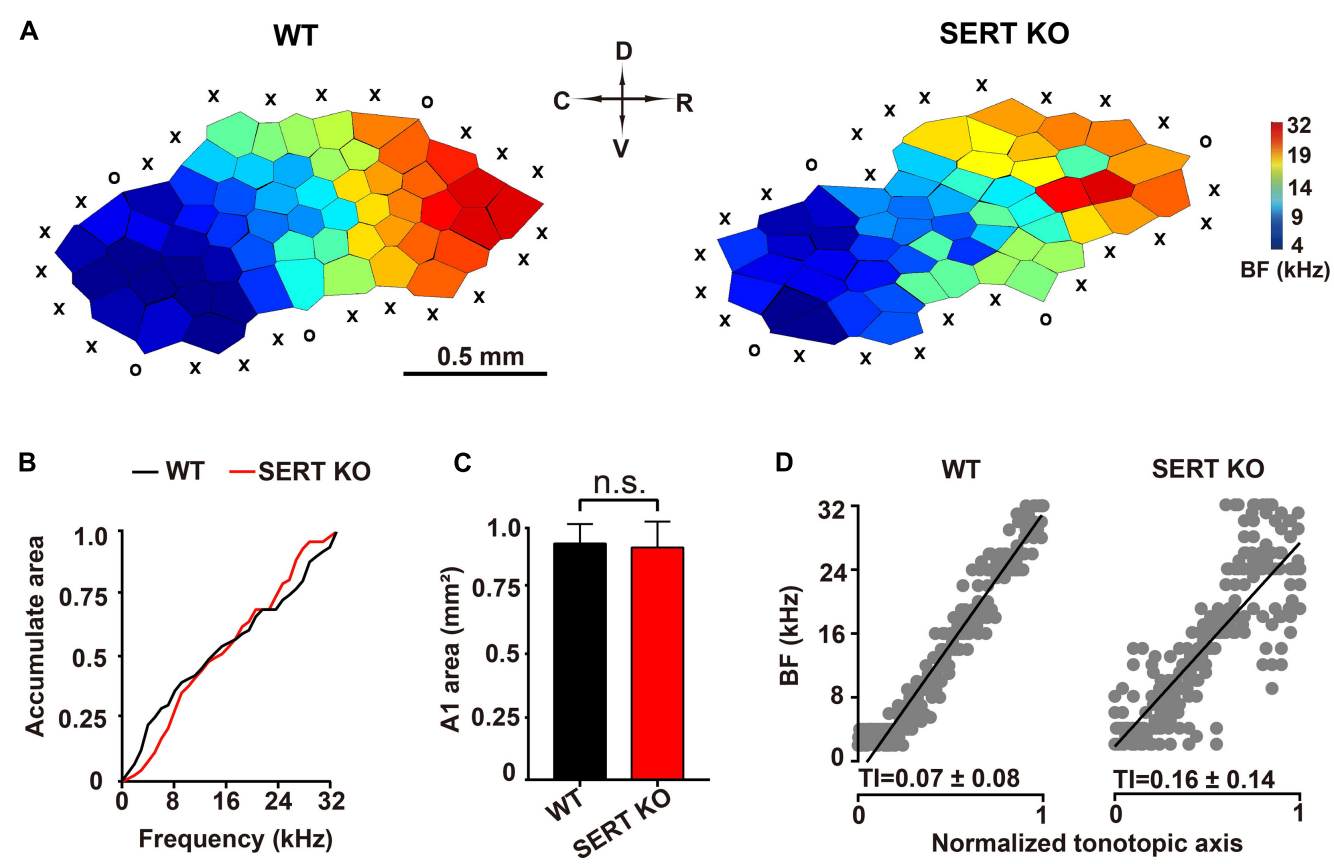

FIGURE 4 | Disturbed tonotopic organization of primary auditory cortex in serotonin transporter (SERT) knockout (KO) mice. (A) Representative A1 characteristic-frequency map from a wild-type (WT) mouse (left) and a SERT KO mouse (right). Scale bar: 0.5 mm. X, unresponsive cortical site; O, non-A1 cortical site (see section "Materials and Methods"). (B) Plotting accumulated response areas at best frequency for neurons of primary auditory cortex in WT and SERT KO mice. (C) The total areas of primary auditory cortex in WT mice and SERT KO mice. (D) The best frequencies of all recorded sites in WT and SERT KO mice were plotted against a normalized tonotopic axis. The tonotopic index (TI) represents the increased scatter of best frequencies around the ideal tonotopic axis (black diagonal line) (see section "Materials and Methods"). Data are presented as means \pm SD. ns, no statistical difference, $p>0.05$. Student's $t$-test.

neurons, including those in the AC, of adult WT mice (Figure 1). These data suggest that the morphological and functional defects observed in AC neurons of SERT KO mice may not

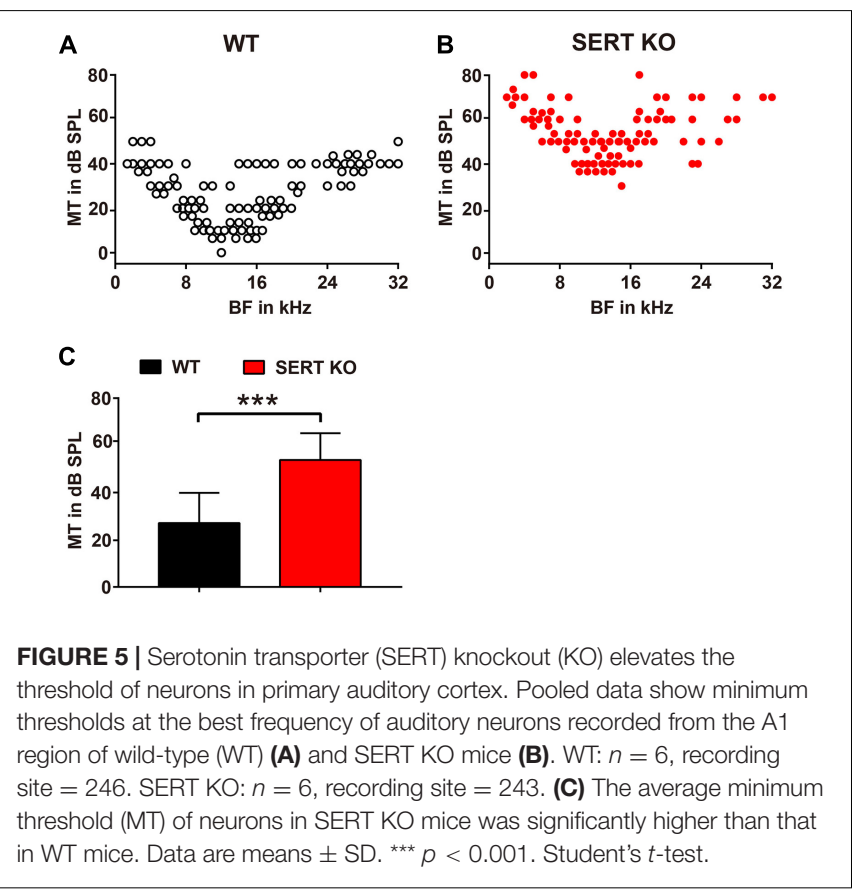

due to the disruption of serotonergic innervations directly but more likely related to the effects of SERT deficit on cortical circuit development.

As the staining results showed, the length of basal dendrites of pyramidal cells was significantly shortened (Figure 2), and the number and density of dendritic spines were significantly reduced in SERT KO mice (Figure 3), suggesting that the ability of pyramidal cells to receive input information was decreased, which might cause less sensitivity to sound response (Figure 5). Moreover, the reduction of the length, the number, and density of dendritic spines in inhibitory interneurons of SERT KO mice suggests that the reduced inhibitory of the interneurons might cause less frequency selectivity of neurons (Figure 6). However, deficient SERT may change the neural morphology in different ways in different brain regions. The length of dendrite and the density of dendritic spines were increased in the infralimbic cortex of SERT KO mice. Meanwhile, SERT KO has little effects on the morphology of basolateral amygdala neurons (Wellman et al., 2007). These different results suggest that the morphology of neurons was also specifically determined by their circuits; even the SERT was abolished in the early stage of the development. Interestingly, many evidences have suggested that SERT is expressed not only on serotonin neurons but also on other neurons such glutamatergic neurons during development. The loss of SERT in these neurons may affect the neural circuit in the cortex during development (Chen et al., 2015). This may explain why the morphology of AC neurons was altered by SERT 


\section{A}

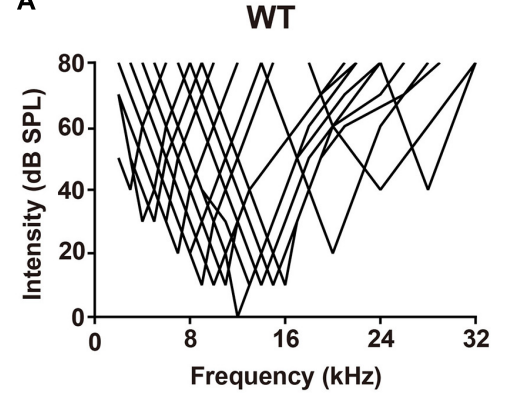

C

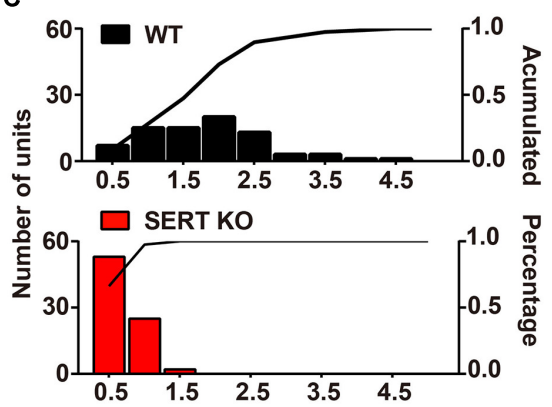

B

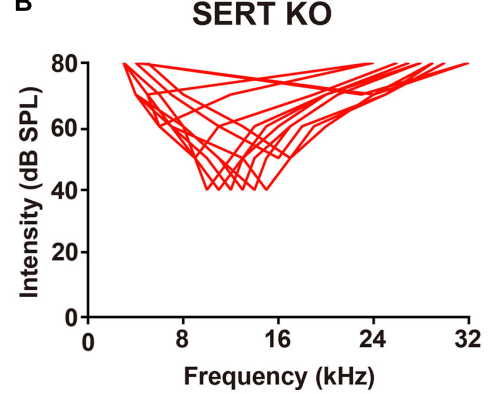

D

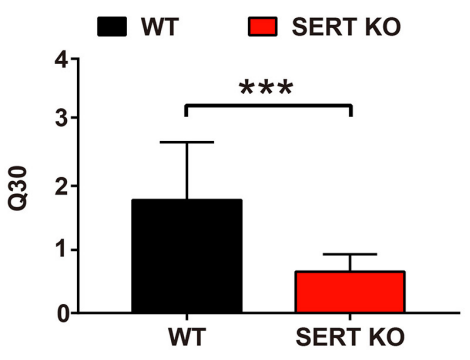

FIGURE 6 | Broader frequency tuning for primary auditory cortical neurons in serotonin transporter (SERT) knockout (KO) mice. Pooled data show the frequency-tuning curves of cortical neurons in wild-type (WT) (A) and SERT KO (B) Al. (C) The distribution of the Q30 values for cortical neurons in WT (top panel) and SERT KO (bottom panel) Al. The lines in the panels represent the cumulative percentage of the Q30 value. (D) The mean Q30 value of all auditory neurons recorded from WT mice and SERT KO Al. Data are presented as means \pm SD. $n=6$ for each group. ${ }^{\star \star \star} p<0.001$. Student's $t$-test.

KO, although few direct serotonergic projections were detected in AC of WT animals.

SERT is a target of SSRIs treatments and regulates the activity of serotonergic system (Kalueff et al., 2009). In the present study, we examined the role of SERT function in primary AC by using SERT KO mice. In WT mice, the primary AC formed a compact and ordered "tonotopic map"; the optimal response frequencies from the rostral to caudal side was regularly distributed. But in SERT KO mice, the primary AC formed a distorted tonotopic organization (Figure 4). However, in SERT KO mice, the disordered frequency topology did not show a systematic decrease in frequency, although the neurons that respond to high frequencies were still mostly medial to the rostral side, and the neurons that respond to low frequencies were still mostly lateral to the caudal. Next, we further analyzed the total area of the tonotopic map in the AI region and the frequency range of the response to sound (Figure 4B). There was no statistical difference between SERT KO mice and WT mice (Figure 4B). It suggests that SERT may mainly affect the orderliness of the neuron's frequency response. Similarly, manipulations of rodent brain serotonin levels during early development, through either increases (produced in SERT or monoamine oxidase KO mice) or decreases (produced by parachlorophenylalanine or other treatments), alter the formation of the whisker (barrel) representation in the primary somatosensory cortex and promote aggressive and/or anxiety-related behaviors (Cases et al., 1995, 1996; Persico et al., 2000; Persico et al., 2001; Holmes et al., 2003; Jennings et al., 2006). In our previous study, auditory mismatch negativity response was found abolished by SERT KO, indicating the low ability in frequency discrimination (Pan et al., 2020). This finding was supported by the functional changes of AC observed in the present study. Under the administration of SSRIs, the animal showed anxiety-related and ASD-like behavior (Simpson et al., 2011; Pan et al., 2020). Although the abnormal behavior and auditory function were quite consistent in SERT KO and SSRI-administered animals, the direct evidence is still very limited for us to understand the effects of SSRIs on development and function of cortical circuits. However, the SERT KO mouse model used in the present study is constitutive and global. To investigate the role of SERT and SSRI in the development of ASD and anxiety, a knock-in SERT mouse model, such as SERT Ala56, should be expected in future studies (Siemann et al., 2017).

One of previous findings related to serotonin and early cortical organization found that the primary sensory cortex of mice, especially the layer IV neurons of visual, auditory, and somatosensory cortex, is transiently innervated with dense 5-HT-containing projection (D'Amato et al., 1987; Blue et al., 1991; Bennett-Clarke et al., 1994). Moreover, in early brain development, 5- $\mathrm{HT}$ is taken up into glutamatergic thalamocortical terminals (Bennett-Clarke et al., 1996; Lebrand, 1996) and used in combination with the 5-HT 1B receptor on layer IV afferents (Salichon et al., 2001). Serotonin plays a trophic role in brain morphogenesis, including cell proliferation, migration, and differentiation. It is also one of the first neurotransmitters to appear in the central nervous system (Azmitia, 2001; Gaspar et al., 2004). We found that the typical 
dendritic morphology was shortened (Figure 2) and that the density of dendritic spines was lower in SERT KO mice compared with WT mice (Figure 3). In SERT KO mice, the deficit of SERT may alter neural morphology and distort tonotopic organization in primary AC.

The structure and arborization of dendrites have a profound impact on the processing of neuronal information because they determine the extent of a neuron's synaptic field. Thousands of spines stud the pyramidal cell's apical and basal dendritic branches, increasing the neuron's receptive surface area and allowing for integration of thousands of excitatory signals to influence the output (Spruston, 2008). Obviously, dendritic spines receive most of the excitatory impulses of a pyramidal cell, consistent with the information processing capacity of the neuron. A cell with spiny processes in homologous nuclei has more spines, the higher level of the subject in the animal series. Thus, as an example in vertebrates, the Purkinje cell of birds shows fewer spines than that of mammals.

Since neural circuits are defined by inter-neuronal communications, output precision in individual cells becomes essential to network function. Cortical interneurons use rhythmic inhibition to create narrow windows for effective excitation, entraining excitatory pyramidal cells to fire certain oscillatory patterns (Beierlein et al., 2000; Uhlhaas and Singer, 2010). Moreover, optogenetic manipulation of specific excitatory and inhibitory circuits directly caused changes in social and cognitive behaviors in mice (Yizhar et al., 2011). Thus, circuits tend to be malleable but vulnerable during post-natal development. Notably, several neurodevelopmental disorders, such as ASD and schizophrenia (SCZ), manifest during this plasticity period (Zoghbi, 2003; Hossein and Folsom, 2009).

Interestingly, in our results, the neuron functions altered; especially, the MT for a BF of SERT KO mice was significantly higher than $40 \mathrm{~dB}$ (Figure 5). It suggested that absent SERT reduced the neurons' intensity sensitivity to response. Moreover, in SERT KO mice, Q30 values were mostly below 1 (Figure 6). Meanwhile, the frequency-tuning curve was dull, and the frequency selectivity of neurons was significantly worse (Figure 6). It suggested that neurons in SERT KO mice showed less frequency selectivity to response.

\section{REFERENCES}

Arellano, J. I., Benavides-Piccione, R., Defelipe, J., and Yuste, R. (2007). Ultrastructure of dendritic spines: correlation between synaptic and spine morphologies. Front. Neurosci. 1:131-143. doi: 10.3389/neuro.01.1.1.010.2007

Azmitia, E. C. (2001). Modern views on an ancient chemical: serotonin effects on cell proliferation, maturation, and apoptosis. Brain Res. Bull. 56, 413-424. doi: 10.1016/S0361-9230(01)00614-1

Bao, S., Chang, E. F., Davis, J. D., Gobeske, K. T., and Merzenich, M. M. (2003). Progressive degradation and subsequent refinement of acoustic representations in the adult auditory cortex. J. Neurosci. 23, 10765-10775. doi: 10.1523/ JNEUROSCI.23-34-10765.2003

Bao, S., Chang, E. F., Woods, J., and Merzenich, M. M. (2004). Temporal plasticity in the primary auditory cortex induced by operant perceptual learning. Nat. Neurosci. 7, 974-981. doi: 10.1038/nn1293

Beierlein, M., Gibson, J. R., and Connors, B. W. (2000). A network of electrically coupled interneurons drives synchronized inhibition in neocortex. Nat. Neurosci. 3, 904-910. doi: 10.1038/78809
All of these results may be related to shortened typical dendritic morphology of neurons (Figure 2) and the lower density of dendritic spines of primary AC in SERT KO mice (Figure 3), which may result in weakened synaptic information transmission between the cortical pyramidal cells and the interneurons of AC in SERT KO mice. The altered function of the primary AC suggests that SERT plays a critical role in circuit development and function.

\section{DATA AVAILABILITY STATEMENT}

The original contributions presented in the study are included in the article/supplementary material, further inquiries can be directed to the corresponding authors.

\section{ETHICS STATEMENT}

The animal study was reviewed and approved by Institutional Animal Care and Use Committee (IACUC) of Southern Medical University.

\section{AUTHOR CONTRIBUTIONS}

HZ and JT designed the experiments. WP, JP, and YZ performed the experiments. All authors wrote, reviewed, edited, and approved the final manuscript.

\section{FUNDING}

This work was supported by the Key-Area Research and Development Program of Guangdong Province (grant number 2018B030331001), the National Natural Science Foundation of China (grant number 81670922), and the Guangdong Basic and Applied Basic Research Foundation (grant number 2020A1515010248).

Bengel, D., Murphy, D. L., Andrews, A. M., Wichems, C. H., Feltner, D., Heils, A., et al. (1998). Altered brain serotonin homeostasis and locomotor insensitivity to 3,4-methylenedioxymethamphetamine ("Ecstasy") in serotonin transporterdeficient mice. Mol. Pharmacol. 53:649. doi: 10.1124/mol.53.4.649

Bennett-Clarke, C. A., Chiaia, N. L., and Rhoades, R. W. (1996). Thalamocortical afferents in rat transiently express high-affinity serotonin uptake sites. Brain Res. 733, 301-306. doi: 10.1016/0006-8993(96)00791-3

Bennett-Clarke, C. A., Leslie, M. J., Lane, R. D., and Rhoades, R. W. (1994). Effect of serotonin depletion on vibrissa-related patterns of thalamic afferents in the rat's somatosensory cortex. J. Neurosci. 14, 7594-7607. doi: 10.1523/JNEUROSCI. 14-12-07594.1994

Blazer, D. G., and Tucci, D. L. (2018). Hearing loss and psychiatric disorders: a review. Psychol. Med. 49, 891-897. doi: 10.1017/S0033291718003409

Blue, M. E., Erzurumlu, R. S., and Jhaveri, S. (1991). A comparison of pattern formation by thalamocortical and serotonergic afferents in the rat barrel field cortex. Cereb. Cortex 1, 380-389. doi: 10.1093/cercor/1.5.380

Cases, O., Seif, I., Grimsby, J., Gaspar, P., Chen, K., Pournin, S., et al. (1995). Aggressive behavior and altered amounts of brain serotonin and 
norepinephrine in mice lacking MAOA. Science 268, 1763-1766. doi: 10.1126/ science. 7792602

Cases, O., Vitalis, T., Seif, I., De Maeyer, E., Sotelo, C., and Gaspar, P. (1996). Lack of barrels in the somatosensory cortex of monoamine oxidase a-deficient mice: role of a serotonin excess during the critical period. Neuron 16, 297-307. doi: 10.1016/S0896-6273(00)80048-3

Chen, X., Ye, R., Gargus, J. J., Blakely, R. D., Dobrenis, K., and Sze, J. Y. (2015). Disruption of transient serotonin accumulation by non-serotonin-producing neurons impairs cortical map development. Cell Rep. 10, 346-358. doi: 10.1016/ j.celrep.2014.12.033

D’Amato, R. J., Blue, M. E., Largent, B. L., Lynch, D. R., Ledbetter, D. J., Molliver, M. E., et al. (1987). Ontogeny of the serotonergic projection to rat neocortex: transient expression of a dense innervation to primary sensory areas. Proc. Natl. Acad. Sci. U.S.A. 84, 4322-4326. doi: 10.1073/pnas.84.12.4322

de Villers-Sidani, E., Chang, E. F., Bao, S., and Merzenich, M. M. (2007). Critical period window for spectral tuning defined in the primary auditory cortex (A1) in the rat. J. Neurosci. 27, 180-189. doi: 10.1523/JNEUROSCI.3227-06.2007

Gaspar, P., Cases, O., and Maroteaux, L. (2004). The developmental role of serotonin: news from mouse molecular genetics. Nat. Rev. Neurosci. 4, 10021012. doi: $10.1038 / \mathrm{nrn} 1256$

Glaser, E. M., and Loos, H. (1981). Analysis of thick brain sections by obversereverse computer microscopy: application of a new, high clarity Golgi-Nissl stain. J. Neurosci. Methods 4, 117-125. doi: 10.1016/0165-0270(81)90045-5

Gray, E. G. (1959). Electron microscopy of synaptic contacts on dendrite spines of the cerebral cortex. Nature 183, 1592-1593. doi: 10.1038/1831592a0

Holmes, A., Yang, R. J., Lesch, K. P., Crawley, J. N., and Murphy, D. L. (2003). Mice lacking the serotonin transporter exhibit 5-HT(1A) receptor-mediated abnormalities in tests for anxiety-like behavior. Neuropsychopharmacology 28, 2077-2088. doi: 10.1038/sj.npp.1300266

Hossein, F. S., and Folsom, T. D. (2009). The neurodevelopmental hypothesis of schizophrenia, revisited. Schizophr. Bull. 35, 528-548. doi: 10.1093/schbul/ $\operatorname{sbn} 187$

Jennings, K. A., Loder, M. K., Sheward, W. J., Pei, Q., Deacon, R. M., Benson, M. A., et al. (2006). Increased expression of the 5-HT transporter confers a low-anxiety phenotype linked to decreased 5-HT transmission. J. Neurosci. 26, 8955-8964. doi: 10.1523/JNEUROSCI.5356-05.2006

Kaas, J. H., Hackett, T. A., and Tramo, M. J. (1999). Auditory processing in primate cerebral cortex. Curr. Opin. Neurobiol. 9, 164-170. doi: 10.1016/S09594388(99)80022- 1

Kalueff, A. V., Olivier, J., Nonkes, L., and Homberg, J. R. (2009). Conserved role for the serotonin transporter gene in rat and mouse neurobehavioral endophenotypes. Neurosci. Biobehav. Rev. 34, 373-386. doi: 10.1016/j. neubiorev.2009.08.003

Kehrle, H. M., Sampaio, A., Granjeiro, R. C., Oliveira, T., and Oliveira, C. A. C. P. (2015). Tinnitus annoyance in normal-hearing individuals: correlation with depression and anxiety. Anna. Otol. Rhinol. Laryngol. 125, 185-194. doi: 10. $1177 / 0003489415606445$

Kelly, J. B., and Sally, S. L. (1988). Organization of auditory cortex in the albino rat: binaural response properties. J. Neurophysiol. 59, 1756-1769. doi: 10.1152/jn. 1988.59.6.1756

Kogoj, A. (2014). Selective serotonin reuptake inhibitors-induced delirium: a case review. Psychiatr. Danub. 26, 277-280.

Lebrand, C. (1996). Transient uptake and storage of serotonin in developing thalamic neurons. Neuron 17, 823-835. doi: 10.1016/S0896-6273(00)80215-9

Pan, W., Lyu, K., Zhang, H., Li, C., and Tang, J. (2020). Attenuation of auditory mismatch negativity in serotonin transporter knockout mice with anxietyrelated behaviors. Behav. Brain Res. 379:112387. doi: 10.1016/j.bbr.2019.112387

Pattyn, T., Van Den Eede, F., Vanneste, S., Cassiers, L., Veltman, D. J., Van De Heyning, P., et al. (2016). Tinnitus and anxiety disorders: a review. Hear. Res. 333, 255-265. doi: 10.1016/j.heares.2015.08.014

Persico, A. M., Ltamura, C. A., Calia, E., Puglisi-Allegra, S., Ventura, R., Lucchese, F., et al. (2000). Serotonin depletion and barrel cortex development: impact of growth impairment vs. serotonin effects on thalamocortical endings. Cereb. Cortex 10, 181-191. doi: 10.1093/cercor/10.2.181

Persico, A. M., Mengual, E., Moessner, R., Hall, S. F., Revay, R. S., Sora, I., et al. (2001). Barrel pattern formation requires serotonin uptake by thalamocortical afferents, and not vesicular monoamine release. J. Neurosci. 21, 6862-6873. doi: 10.1523/JNEUROSCI.21-17-06862.2001
Salichon, N., Gaspar, P., Upton, A. L., Picaud, S., Hanoun, N., Hamon, M., et al. (2001). Excessive activation of serotonin (5-HT) 1B receptors disrupts the formation of sensory maps in monoamine oxidase A and 5-HT transporter knock-out mice. J. Neurosci. 21, 884-896. doi: 10.1523/JNEUROSCI.21-0300884.2001

Sholl, D. A. (1953). Dendnitic organization in the neurons of the visual and motor cortices of the cat. J. Anat. 87, 387-406. doi: 10.1038/171387a0

Siemann, J. K., Muller, C. L., Forsberg, C. G., Blakely, R. D., Veenstra-VanderWeele, J., and Wallace, M. T. (2017). An autism-associated serotonin transporter variant disrupts multisensory processing. Transl. Psychiatry 7:e1067. doi: 10. 1038/tp.2017.17

Simpson, K. J., Weaver, K. J., de Villers-Sidani, E., Lu, J. Y. F., Cai, Z., Pang, Y., et al. (2011). Perinatal antidepressant exposure alters cortical network function in rodents. Proc. Natl. Acad. Sci. U.S.A. 108, 18465-18470.

Spacek, J., and Harris, K. M. (1998). Three-dimensional organization of cell adhesion junctions at synapses and dendritic spines in area CAl of the rat hippocampus. J. Comp. Neurol. 393, 58-68.

Spruston, N. (2008). Pyramidal neurons: dendritic structure and synaptic integration. Nat. Rev. Neurosci. 9, 206-221. doi: 10.1038/nrn2286

Stiebler, I., Neulist, R., Fichtel, I., and Ehret, G. (1997). The auditory cortex of the house mouse: left-right differences, tonotopic organization and quantitative analysis of frequency representation. J. Compar. Physiol. A 181, 559-571.

Tang, J., and Suga, N. (2009). Corticocortical interactions between and within three cortical auditory areas specialized for time-domain signal processing. J. Neurosci. 29, 7230-7237.

Tang, J., Xiao, Z. J., and Shen, J. X. (2008). Delayed inhibition creates amplitude tuning of mouse inferior collicular neurons. Neuroreport 19, 1445-1449.

Tang, J., Yang, W., and Suga, N. (2012). Modulation of thalamic auditory neurons by the primary auditory cortex. J. Neurophysiol. 108, 935-942.

Uhlhaas, P. J., and Singer, W. (2010). Abnormal neural oscillations and synchrony in schizophrenia. Nat. Rev. Neurosci. 11, 100-113.

Umbricht, D., Vyssotki, D., Latanov, A., Nitsch, R., and Lipp, H. P. (2005). Deviance-related electrophysiological activity in mice: is there mismatch negativity in mice? Clin. Neurophysiol. 116, 353-363.

Wellman, C. L., Izquierdo, A., Garrett, J. E., Martin, K. P., Carroll, J., Millstein, R., et al. (2007). Impaired stress-coping and fear extinction and abnormal corticolimbic morphology in serotonin transporter knock-out mice. J. Neurosci. 27, 684-691.

Yizhar, O., Fenno, L. E., Prigge, M., Schneider, F., Davidson, T. J., O’Shea, D. J., et al. (2011). Neocortical excitation/inhibition balance in information processing and social dysfunction. Nature 477, 171-178.

Yuste, R., and Denk, W. (1995). Dendritic spines as basic units of synaptic integration. Nature 375, 682-684. doi: 10.1038/375682a0

Zhang, L., Wang, B., Zhang, L., Wang, J., Zhang, M., Cui, M., et al. (2009). A modified Golgi-Cox staining method for study the structure and morphology of neurons in the striatum. Chin. J. Neuroanat. 25, 343-347.

Zhang, L. I., Bao, S., and Merzenich, M. M. (2001). Persistent and specific influences of early acoustic environments on primary auditory cortex. Nat. Neurosci. 4, 1123-1130. doi: 10.1038/nn745

Zoghbi, H. Y. (2003). Postnatal neurodevelopmental disorders: meeting at the synapse? Science 302, 826-830. doi: 10.1126/science.1089071

Conflict of Interest: The authors declare that the research was conducted in the absence of any commercial or financial relationships that could be construed as a potential conflict of interest.

Publisher's Note: All claims expressed in this article are solely those of the authors and do not necessarily represent those of their affiliated organizations, or those of the publisher, the editors and the reviewers. Any product that may be evaluated in this article, or claim that may be made by its manufacturer, is not guaranteed or endorsed by the publisher.

Copyright (®) 2021 Pan, Pan, Zhao, Zhang and Tang. This is an open-access article distributed under the terms of the Creative Commons Attribution License (CC BY). The use, distribution or reproduction in other forums is permitted, provided the original author(s) and the copyright owner(s) are credited and that the original publication in this journal is cited, in accordance with accepted academic practice. No use, distribution or reproduction is permitted which does not comply with these terms. 